\title{
TB1 shapes inflorescence
}

Plant Cell 30, 563-581 (2018)

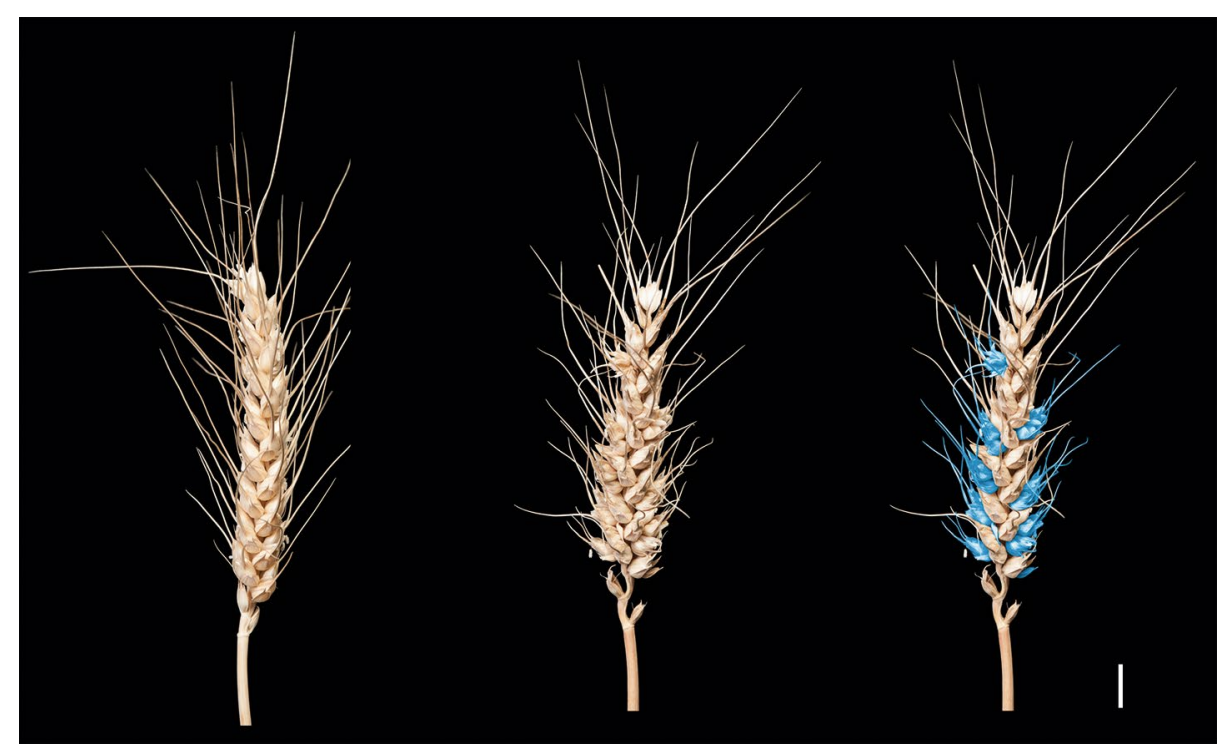

Credit: Andrew Davis and Scott Boden, John Innes Centre

Grass inflorescence consists of many flowers arranged on reproductive branches, known as spikelets, and this architecture largely determines the yield. Bread wheat, one of the most important staple crops, safeguards world food security but the genetic basis controlling its inflorescence development remains enigmatic. To address this question, Laura E. Dixon, from the John Innes Centre, UK, and colleagues focused on a supernumerary-spikelet trait known as paired spikelets. They found that increased dosage of TEOSINTE BRANCHED1 (TB1) controls this trait.

A pair of near-isogenic lines showed either wild-type inflorescences or inflorescences with paired spikelets. Compared to the wild type (pictured to the left), the line with paired spikelets (pictured right and centre) exhibited a delay in inflorescence development, producing more spikelets and fewer tillers. Genetic analysis showed that paired spikelet is a semidominant trait affected by the dosage of chromosome 4D.

Consistently, it was previously identified that a quantitative trait locus (QTL) on chromosome $4 \mathrm{D}$ regulates paired spikelet development. Examination of the QTL yielded a candidate gene, a wheat homologue of the well-known maize domestication gene, TB1. The line with paired spikelets has doubled copies of TB1 and significantly higher TB1 expression in developing inflorescences and emerging tillers. Transgenic experiments confirmed that higher dosage of TB1 promotes paired spikelet development and suppresses tiller outgrowth.

TB1 interacts with the FLOWERING

LOCUS T1 (FT), which is known to promote expression of spikelet meristem identity genes. In plants with paired spikelets, higher expression of TB1 was associated with reduced expression of meristem identity genes. So increased TB1 expression probably suppresses meristem identity genes by reducing the effect of FT, which consequently facilitates the development of paired spikelets.

Natural variations for TB1 regulate paired spikelet development in modern cultivars, and could potentially facilitate the breeding of high-yield wheat.

\section{Jun Lyu}

Published online: 23 April 2018

https://doi.org/10.1038/s41477-018-0155-4 